\title{
El apoyo del gobierno como deter- minante de la colaboración exitosa entre la universidad y la empresa*
}

Government Support as a Determinant of Successful University-Enterprise Collaboration

$\bigcirc$ apoio do Governo como determinante da colaboração de sucesso entre a universidade

e a empresa

\author{
Gilberto Parra Gaviño \\ Rosa Amalia Gómez Ortiz \\ Isidoro Pastor Román ${ }^{\star \star \star \star}$
}

Fecha de recibido: 9 de diciembre de 2014

Fecha de aprobado: 1 de abril de 2015

Doi: dx.doi.org/10.12804/rev.univ.empresa.29.2015.09

Para citar este artículo: Parra Gaviño, G., Gómez Ortiz, R. A., \& Pastor Román, I. (2015). El apoyo del gobierno como determinante de la colaboración exitosa entre la universidad y la empresa. Universidad \& Empresa, 17(29), 213-238. Doi: dx.doi.org/10.12804/rev.univ.empresa.29.2015.09

* Este artículo es resultado del proyecto de investigación titulado "Experiencias de colaboración universidad-empresa-Gobierno en diferentes países", el cual fue financiado por el Instituto Politécnico Nacional (IPN) (Distrito Federal, México). El objetivo general fue identificar los elementos que propician una mayor colaboración entre la universidad y la empresa (CUE), con base en la experiencia de otros países

** Estudiante del Doctorado en Ciencias Administrativas de la Escuela Superior de Comercio y Administración del Instituto Politécnico Nacional (IPN) (Distrito Federal, México). Profesor-investigador en esta misma institución. Correo electrónico: mtro.gilbertoparra@hotmail.com

*** Doctora en Ciencias Administrativas de la Escuela Superior de Comercio y Administración del Instituto Politécnico Nacional (IPN) (Distrito Federal, México). Profesora-investigadora en esta misma institución. Correo electrónico: ragomez100@hotmail.com

***** Doctor en Ciencias Administrativas de la Escuela Superior de Comercio y Administración del Instituto Politécnico Nacional (IPN) (Distrito Federal, México). Profesor-investigador en esta misma institución. Correo electrónico: pastorroman@hotmail.com 


\section{RESUMEN}

La colaboración entre la universidad y la empresa -CUE- es un mecanismo necesario para que se dé la transferencia de conocimiento entre una y otra. En esta investigación se analizan los principales problemas que enfrenta esta relación y el papel que ha desempeñado el gobierno, un actor que es identificado en el estudio como fundamental, considerando lo encontrado en varios países. Ello se hace con el fin de identificar qué elementos deben considerarse para fortalecer este esquema cooperativo. Para lograr dicho objetivo se realizó un análisis comparativo de las contribuciones de múltiples autores relevantes. Gracias a esto fue posible encontrar diez elementos que deben considerarse para fortalecer la CUE, siendo el de mayor relevancia, entre ellos, la revisión del marco legal e institucional.

Palabras clave: articulación universidad-empresa-gobierno, colaboración universidad-empresa, gobierno facilitador.

\section{ABSTRACT}

The University-Enterprise Collaboration -UEC- is a mechanism required to transfer knowledge between the two instances. In this research, the main problems faced by this relationship and the role of the government, an actor identified in the study as fundamental in view of what has been found in several countries, are analyzed. This is undertaken in order to identify which elements must be considered in order to strengthen this cooperative scheme. To achieve this objective, the contributions of many important authors were subjected to a comparative analysis. This made it possible to identify the ten elements that have to be considered in order to strengthen the UEC. The most important element of all is revision of the legal and institutional framework.

Keywords: University-enterprise-government articulation, university-enterprise collaboration, facilitating government.

\section{RESUMO}

A colaboração entre a universidade e a empresa (CUE) é um mecanismo necessário para que se dê a transferência de conhecimento entre uma e outra. Nesta pesquisa se analisam os principais problemas que enfrenta esta relação e o papel que tem desempenhado o Governo, um ator que é identificado no estudo como fundamental, considerando o encontrado em vários países. Isto se faz com o fim de identificar quais elementos devem se considerar para fortalecer este esquema cooperativo. Para conseguir este objetivo, realizou-se uma análise comparativo das contribuições de múltiplos autores relevantes. Graças a isto foi possível encontrar dez elementos que devem considerar-se para fortalecer a CUE, sendo o de maior relevância, entre eles, a revisão do marco legal e institucional.

Palavras-chave: articulação universidade-empresa-Governo, colaboração universidadeempresa, Governo facilitador. 


\section{INTRODUCCIÓN}

Un concepto que en el ámbito económico mundial ha evolucionado rápidamente en las últimas cuatro décadas y que sigue generando mucha discusión, es la colaboración universidad-empresa-CUE- (Ramírez Salazar \& García Valderrama, 2010). Esto no solo porque la globalización ha forzado a las empresas a ser cada vez más competitivas (Hitt, Ireland, \& Hoskisson, 2013) $\mathrm{y}$, entre otras acciones vinculadas con este hecho, a llevar a cabo proyectos conjuntos de investigación y desarrollo (I+D) con las universidades, sino también porque dichas instituciones académicas han tenido que adaptarse a los cambiantes requerimientos del sector productivo (Deem, Hillyard, \& Reed, 2007; Jiménez \& Ramos, 2009; National Research Council, 2012).

El papel de los gobiernos es determinante para iniciar y sostener dicha colaboración, pero también lo es la disposición de las universidades a través de iniciativas concretas que están a su disposición en la actualidad (Carballo Peña, Rodríguez Silva, \& García Arias, 2008; Gibbons et al., 1994; McCaffery, 2004). Entre estas, Bhattachayra y Arora (2007) resaltan, por ejemplo, la posibilidad de crear centros especiales para facilitar la interacción con la industria. No obstante, es preciso considerar que la cuE se sitúa en diferentes contextos y coyunturas que conllevan diferentes acciones que se relacionan con otros conceptos como la investigación científica, el desarrollo y la innovación tecnológica. Esta varía en función de múltiples factores y circunstancias, como el nivel de desarrollo de los países, el tipo de industrias (maduras o emergentes) con las que se cuenta, las universidades existentes (tradicionales o de clase mundial), los sistemas de innovación, el apoyo de los gobiernos, la disposición de las empresas para participar e, incluso, entre otros factores, los recursos financieros disponibles y los que están en juego en cada decisión respecto de esta relación y sus posibles frutos (Viale \& Etzkowitz, 2010).

Los dirigentes de economías industrializadas y de algunas emergentes, a través de sus organizaciones gubernamentales e instancias relacionadas con la ciencia y la tecnología, han estado concentrados en fomentar la interacción entre la ciencia y la industria (Martínez \& Fundación IKERTIA, 2007), así como en diseñar políticas destinadas a aumentar la calidad de los centros de investigación pública y de las instituciones educativas (Etzkowitz, 2008). Esto con el fin de desarrollar un mejor y más productivo emprendimiento $\mathrm{y}$ 
generar así mayores beneficios para el desarrollo nacional (Carayannis \& Campbell, 2012; Nowotny, Scott, \& Gibbons, 2001). Se busca además incrementar la capacidad de estas instituciones para apoyar de manera más decidida y efectiva a las empresas, para que estas, en la medida de lo posible, lleguen a realizar actividades tecnológicas más dinámicas, avanzadas y que representen un mayor valor agregado (Bodas Freitas, Argou, \& Mirra de Paula, 2013).
La figura 1 expone los principales elementos que caracterizan la CuE. Se señalan, entre otros aspectos, el papel que juega el gobierno en el diseño de políticas públicas y en el fomento de la interacción entre la ciencia y la industria. Esto en el marco de una globalización que empuja a las organizaciones a ser cada vez más competitivas y a las universidades a respaldar de manera creciente al sector productivo.

Figura 1. Caracterización de la CUE

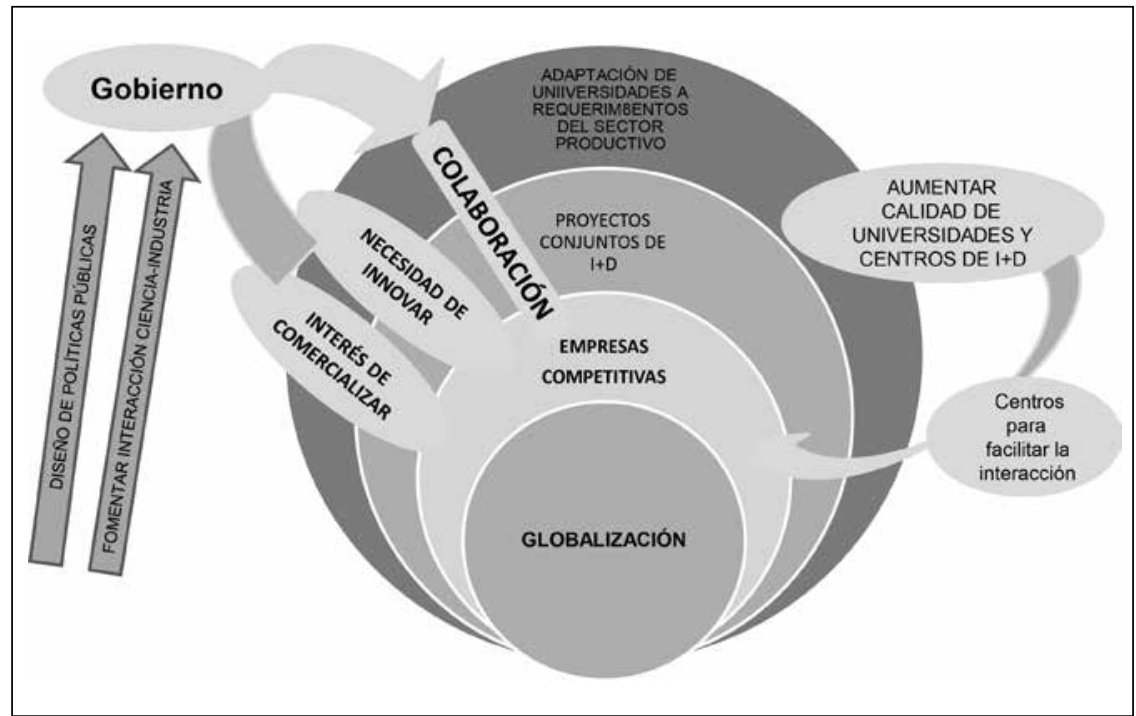

Fuente: Elaboración propia.

Los tres actores aludidos, universidad, empresas y gobierno, deben articularse (Etzkowitz, 2008). El éxito de la CUE, sin embargo, no solo depende de las acciones que realicen cada uno de estos, sino también de múltiples otros factores y circunstancias no necesariamente vinculados de manera directa con ellos, pero que sí pueden afectarlos. 
Considerando en particular la importancia de esta relación para la sociedad actual (Viale \& Etzkowitz, 2010), el objetivo de esta investigación fue presentar resultados desde una perspectiva analítica de los elementos que propician una mayor CUE. Esto a partir de la consideración del papel que le corresponde a las universidades, las empresas $y$, especialmente, al gobierno, a la hora de impulsar y desarrollar de manera adecuada este tipo de procesos.

La pregunta de investigación que orientó el desarrollo de este trabajo fue: ¿Qué elementos deben considerar los gobiernos para fortalecer la colaboración universidad-empresa? Para responderla se realizó una revisión de la literatura y un análisis comparativo de las principales contribuciones de autores relevantes para el tema. Esto permitió identificar diez elementos que deben considerarse para fortalecer la CUE, siendo el de mayor relevancia, entre ellos, la revisión del marco legal e institucional.

El presente texto incorpora los hallazgos de la revisión de literatura realizada, abordando tanto los antecedentes de la CUE como su importancia, factores de incidencia, consideraciones sobre el concepto y el papel del gobierno. El documento también expone los elementos metodológicos básicos que permitieron la realización del trabajo y presenta una síntesis de sus resultados esenciales, una discusión de los mismos y las principales conclusiones a las que fue posible llegar gracias al estudio.

\section{REVISIÓN DE LA LITERATURA}

\subsection{Antecedentes}

Existe evidencia de colaboraciones entre empresas europeas e investigadores de universidades en el siglo XIX, sin embargo, experiencias más explícitas se presentaron en la antigua Unión Soviética, en donde las universidades tenían estrechos vínculos con las empresas que eran propiedad del Estado, de ahí que estos lazos fueran establecidos y controlados por el gobierno (Smirnova, 2014). China aprendió de la Unión Soviética y desarrolló una estructura que controlaba, a través de una variedad de ministerios, las actividades productivas, educativas y de investigación. Si bien está realidad ha cambiado desde que China decidió en 1978 participar en la competencia internacional y más tarde incorporarse a la Organización Mundial de Comercio (OMC), subsiste el hecho de que muchas universidades y empresas son administradas por dependencias gubernamentales. De acuerdo con Hong \& Su (2013), esto facilita la proximidad en un 
país con un territorio tan extenso y atenúa así algunos de los efectos negativos derivados del distanciamiento geográfico.

Las universidades y las empresas han mantenido relaciones no formales en particular desde la segunda mitad del siglo $\mathrm{xx}$, aunque han trabajado juntos en temas de pasantía y programas de grado. Mead et al. (1999), sin embargo, agregan otras formas de colaboración más formal en materia de formación profesional, ingeniería y tecnologías, programas de posgrado, foros y eventos, aunque los autores se hacen preguntas en torno a los beneficios de la colaboración, al propio proceso y a su gestión exitosa. Esto lleva a considerar cuestiones como por qué colaborar, cuáles son los beneficios y cómo propiciar una colaboración exitosa entre los actores.

Desde principios del siglo XXI existe una gran voluntad de participar en actividades sustantivas como la transferencia de tecnología, vía la CUE. Es en realidad por la consideración de este nuevo momento que autores como Lai (2011) la consideran más bien un fenómeno reciente, que busca cerrar la brecha entre la industria y la academia, lo que está relacionado con el hecho de que múltiples gobiernos estén destinando porciones cada vez más importantes de su presupuesto a la investigación y el desarrollo (I+D). Ello con el fin de elevar los estándares de la industria y mejorar la competitividad nacional. Para ello han modificado en particular diversas leyes y reglamentos que inciden en esta colaboración. Este autor añade que Japón y Taiwán, por ejemplo, han creado institutos para la transferencia de tecnología, buscado que un creciente número de doctores trabaje en las universidades, a fin de competir, en lo fundamental, con Estados Unidos. Este país, de hecho, recuperó su posición en los mercados por la vía de, entre otras muchas acciones, iniciativas como la Ley Bayh-Dole (Bayh-Dole Act o Patent and Trademark Law Amendments Act), la cual construyó múltiples puentes entre las empresas y las universidades.

En los noventa, Japón le dio un fuerte impulsó a la CUE. Lo hizo con el fin de enfrentar el posicionamiento norteamericano en sectores como la biotecnología, lo que constituyó un cambio de tendencias para un país acostumbrado a estampar su huella en la industria manufacturera (Liew, Tengku \& Lim, 2013). Hechos como este, condujeron a considerar que la CUE desempeñaba, en efecto, un papel trascendente y que impulsarla significaría para Japón - como en efecto lo fue- un giro al timón, el cual le ha permitido competir de 
manera relevante en el terreno industrial y ampliar sus cuotas en el mercado, tal como lo hizo Estados Unidos en los años ochenta, por la vía de una serie de disposiciones gubernamentales, como la indicada en el párrafo anterior, así como la definición de metas específicas en materia de dicha colaboración.

La actual es la era de la economía del conocimiento (Böhme \& Stehr, 1986; UNESCO, 2005). Esta se caracteriza, entre otros aspectos, por la velocidad con que se presentan los cambios tecnológicos, de ahí que, en el marco de la CUE, temas como la transferencia de tecnología, la acumulación de conocimiento, la innovación y la construcción de derechos de propiedad intelectual estén cobrando una creciente importancia. Aunque estos asuntos son más complejos de instrumentar en regiones y países en desarrollo debido, entre otros factores, al desconocimiento que se tiene en algunos de sus ámbitos, incluyendo el gubernamental (Saad \& Zawdie, 2011). A pesar de lo anterior, autores como Hong \& $\mathrm{Su}$ (2013) consideran que la CUE es uno de los mecanismos clave para la transferencia de conocimiento de la universidad a la empresa y, por medio de ella, del desarrollo de los países. En este mismo sentido, Muscio, Quaglione \& Scarpinato (2012) afirman que existe una creciente conciencia, derivada de lo anterior, de que la CUE facilita en efecto la transferencia de conocimiento y que es un potente motor de innovación y desarrollo. Esto contrasta con la visión que se tenía de las universidades en el pasado, cuyo papel se limitaba frecuentemente a transmitir conocimientos a los nuevos profesionales $\mathrm{y}$, en lo fundamental, a apoyar el desarrollo regional (Mockus, 2012).

\subsection{Importancia de la CUE}

Para entender la importancia de la CUE es preciso tomar en cuenta, entre otras cosas, que el conocimiento se considera un recurso vital para la prosperidad de la sociedad (Drucker, 1996). Se considera, de hecho, que el poder que tienen algunas economías en la actualidad, se deriva en buena medida de sus capacidades para creación de conocimiento (Nonaka \& Takeuchi, 1995), por ello se les conoce como economías basadas en conocimiento (Khan \& Park, 2012; Organisation for Economic Co-Operation and Development OECD-, 1996; Schilirò, 2012).

En dicho contexto, la importancia de las universidades radica en que ofrecen a las empresas conocimientos científicos esenciales para sus actividades de innovación, así como soporte técnico y acceso a diversos servicios (Muscio \& Nardone, 2012; 
Muscio et al., 2012). Las empresas dependen cada vez más del conocimiento (Nonaka \& Takeuchi, 2001), lo que está generando fuertes incentivos para desarrollar maneras eficientes de transferir a las mismas los descubrimientos en el ámbito académico (Patthareeya \& Tippawan, 2012; Schwartzman, 2008). Sin embargo, esto varía en la medida en que las universidades están involucradas en la comercialización de sus investigaciones y depende de los sistemas de innovación pues, entre más acceso exista a redes de investigación y conocimiento, que incluyan tecnologías e innovaciones desarrolladas en otras latitudes, mayor impacto tendrán las universidades y un más elevado beneficio tendrán las empresas (Muscio et al., 2012).

El papel de la investigación en las universidades se ha enfatizado fuertemente, ya que muchas buscan convertirse en "universidades de clase mundial" (Salmi, 2009; Wang, Cheng, $\&$ Liu, 2013). Esto para distinguirse de aquellas que se dedican principalmente a enseñar. De hecho, en la búsqueda por definir qué es lo mejor hacia el futuro parece haber una clara preferencia, marcada en particular en las últimas décadas, por privilegiar la investigación (Geiger, 1993; Noll, 1998; Teichler \& Arimoto, 2014). Sin embargo, esto depende en buena medida de los incentivos que reciben las instituciones en cada contexto y de los cambios que estas deciden o no llevar a cabo, pues no es fácil para muchas de ellas redireccionar sus funciones y conseguir el respaldo necesario, por ejemplo, en materia de profesorado.

De acuerdo con Teichler \& Arimoto (2014) la mencionada preferencia varía en función de los países y no tanto de las universidades, en donde, en algunos casos, se habla de "universidades de investigación" (Geiger, 1993). Esto refleja una nueva orientación de las instituciones. Aunque al respecto no es fácil encontrar una tipología claramente consistente y de amplia aceptación, lo que se ha podido identificar es que existen tres tipos de países:

1. En donde hay un claro dominio de la investigación (como en Alemania).

2. En donde existe un balance entre la enseñanza y la investigación (en los países anglosajones).

3. En donde - aunque esto pueda estar cambiando- predomina la enseñanza (como en los países de América Latina).

Es bastante visible que concentrarse solo en la tradicional educación académica y vocacional no es una alternativa efectiva, en particular en una era como la actual, cuando 
se demandan cada vez más amplias y complejas habilidades y competencias al capital humano. Al respecto, Lee (2012) sugiere que las universidades requieren contribuir a cultivar talentos multifacéticos con competencias multifuncionales, esto dependiendo de las necesidades de diversos sectores económicos, en especial de aquellos centrados en la innovación. Por esta razón, la cuE ha adquirido una creciente importancia.

Desde un enfoque tecnológico, en efecto, la recombinación que resulta de la CUE incrementa la posibilidad de mayores rendimientos. Esto se refleja, por ejemplo, en el número de patentes generadas, aunque, a juicio de Soh \& Subramanian (2014), si el foco se pone en la investigación científica, se debilita la relación, ya que las empresas buscan resultados inmediatos que se traduzcan en productos que lleguen fácilmente al mercado. De hecho, no se le ha dado suficiente atención a cómo las empresas acceden al conocimiento científico y en qué medida este es producto o no de descubrimientos realizados en las universidades en el marco de proyectos de CUE.

De acuerdo con Motohashi \& $\mathrm{Mu}-$ ramatsu (2012), es importante que las actividades de las universidades no se limiten a las aplicaciones de la industria, pues un abandono de la in- vestigación básica puede disminuir la calidad de las patentes y reducir los incentivos para las empresas en cuanto a la comercialización de las invenciones resultantes de la colaboración mutua. Por ello es necesario, en particular, 1) analizar todo lo relacionado con el tema de patentes, 2) comparar qué han hecho otros países en materia de leyes e iniciativas gubernamentales y 3 ) evaluar cómo estas acciones han transformado el papel de las universidades en el contexto socioeconómico.

Las universidades actúan como habilitadoras o como conducto para acceder al conocimiento que se produce a nivel internacional, es decir, acercan las empresas a un conjunto de tecnologías e innovaciones desarrolladas en otras latitudes. Ello por su interacción con redes de investigación. Estas instituciones fomentan, además, de múltiples formas el patentamiento. Así mismo, ellas permiten catalizar talentos y facilitar la transferencia, en particular, de conocimiento tácito (Göranzon, Hammaren, \& Ennals, 2006), que es considerado un componente crucial del proceso de innovación (Von Krogh, Ichijō, \& Nonaka, 2000).

Por su parte, la CUE es un elemento básico para el desarrollo de la ciencia y la tecnología, que adopta diversas formas y niveles de compromiso, y 
la investigación es vista como clave para el progreso de las universidades, ya que les proporciona una plataforma para la transferencia de tecnología, revitaliza las actividades académicas y mejora los planes de estudio, lo que constituye una posibilidad para el desarrollo sostenible de la investigación (Liew et al., 2013).

A pesar de dichas ventajas y beneficios, existen limitaciones para medir la CUE. Liew et al. (2013), en particular, proponen usar como parámetro la "aplicabilidad", lo que implica la necesidad de que se generen usos industriales concretos. Estos autores señalan que no basta que se registren propiedades intelectuales si esto no impacta de algún modo en la productividad o la eficiencia de las empresas. Se requiere observar, entonces, en su concepto, qué tanto se logra este objetivo mediante la transferencia de tecnología o el aprovechamiento de conocimientos técnicos.

Aunque algunos investigadores, como Motohashi y Muramatsu (2012), han examinado la CUE en función de aspectos explícitos, es decir, patentes, publicaciones, licencias o ingresos derivados por transferencia de tecnología, este tipo de colaboración va más allá de resultados cuantificables en el corto plazo. Es necesario identificar el común denominador entre universidad e industria, pues permite desarrollar estrategias a largo plazo, las cuales se pueden convertir en una verdadera plataforma para múltiples proyectos. Luego viene el aspecto táctico, que incluye la planeación detallada y la gestión de la colaboración.

Según Liew, Tengku \& Lim (2012), la CUE puede adoptar distintas formas, desde prácticas profesionales o publicaciones, hasta proyectos sofisticados y consultorías de investigación más holísticas. El éxito de estas iniciativas depende, en lo fundamental, de los parámetros utilizados para realizarlas y medirlas. Si bien muchos estudios se centran en aspectos explícitos, hay otros elementos de naturaleza más tácita, más informales, que pueden tener el mismo peso o uno mayor en la mencionada colaboración y que contrastan con la comprensión teórica convencional.

En relación con esto, Motoyama (2014), por ejemplo, examina dos casos en el sector de nanotecnología. Estos revelan la profundidad de la CUE, ya que abarcan diversas disciplinas del conocimiento; se muestra en el trabajo cómo se convierte a las universidades en verdaderos centros para el desarrollo de redes de investigación que brindan la oportunidad a investigadores corporativos de nutrirse del pensamiento teórico de la 
academia y de su tradición. Esto, por supuesto, va más allá de la transferencia de tecnología y de los asuntos de propiedad intelectual.

Motoyama (2014), por su parte, documenta las limitaciones que tienen los investigadores de grandes corporaciones quienes, ante presiones del mercado, se ven obligados a encontrar soluciones inmediatas. Por esta razón no pueden darse tiempo para diseñar preguntas de investigación ni profundizar en un problema, tal como ocurre en el ámbito académico propio de las universidades. De ahí que aprender a pensar diferente e intercambiar experiencias constituya, en realidad, un importante logro para ambas partes. Este puede facilitar, a la larga, el desarrollo de innovaciones radicales o de mayor peso y, por ende, generar un valioso impacto y aprovechamiento que puede prolongarse y derivar nuevas y, en ocasiones, sorprendentes aplicaciones.

Bodas Freitas et al. (2013) identificaron los objetivos más comunes de la CUE. En orden de importancia estos son: el desarrollo de un nuevo producto, un nuevo proceso, el uso de equipos, la capacitación de empleados, mejorar procesos y mejorar productos. Mejorar el conocimiento de la industria y prestar servicios para alcanzar dichos objetivos, sin embargo, no es algo que se pueda lograr fácilmente. Para ello es necesario generan incentivos a los investigadores, así como diseñar políticas públicas, modificar las leyes para establecer reglas en temas tan sensibles como la propiedad intelectual e incentivar las tareas de investigación mediante apoyos financieros e institucionales. La falta de claridad en las leyes, o cualquier otra restricción en estos asuntos, puede generar barreras importantes para el adecuado desarrollo de la CUE. De hecho, en este sentido, se ha logrado identificar también una multiplicidad de barreras y obstáculos adicionales en esta materia, aunque también diversas estrategias que pueden mitigarlas y viabilizar la colaboración, así como una serie de elementos motivadores tanto para las universidades como para las empresas.

\subsection{Factores de incidencia}

La mayoría de los estudios sobre la CUE hablan de sus ventajas, pero pocos han investigado la naturaleza de los obstáculos y los factores que podrían mitigarlos. Bruneel, D'Este \& Salter (2010) examinan dos tipos de barreras: 1) las relacionadas con las diferencias en las orientaciones de la industria y de las universidades y 2) las vinculadas a conflictos sobre propiedad intelectual y cómo tratarlos con las universidades, toda vez que existen diferentes normas 
institucionales que rigen el conocimiento público y privado.

En los últimos treinta años ha crecido la importancia de las universidades en tanto actores económicos (Etzkowitz \& Leydesdorff, 1997; Temple, 2012). El surgimiento de las oficinas de transferencia tecnológica, entre otras iniciativas, ha incrementado su interacción con las empresas. También ha sido de gran ayuda para reducir las barreras a la CUE, la experiencia previa de investigación colaborativa y la confianza mutua. Desde hace tiempo parece haber coincidencia entre múltiples autores acerca de que dos factores de éxito de la colaboración son la confianza y los contactos previos (Barnes, Pashby, \& Gibbons, 2002).

Respecto de las diferencias culturales entre empresas y universidades, Bjerregaard (2010) sugiere la participación de un intermediario que pueda conciliar intereses para generar confianza. Aunque un hallazgo básico de su estudio es que muchos investigadores y colaboradores experimentaron una convergencia institucional, esto es, un espacio cultural para compartir conocimientos en sus proyectos conjuntos. Cuando surge un conflicto normativo, al desdibujarse las lógicas institucionales que rigen la $\mathrm{I}+\mathrm{D}$ en ambos sectores, algunos investigadores logran ser capaces de utilizar sus habilidades sociales para superar estas lagunas.

Otro factor determinante es el interés que muestran hoy académicos, políticos y líderes de la industria por estas temáticas. Se considera, en general, que la cuE tiene un gran valor para la innovación, ya que una estructura tecnológica robusta propicia sinergias y agrupa y acumula potencialidades. Esto permite atender proyectos a través de programas de apoyo y mediante la creación de infraestructura en I+D para las áreas industriales. Además, esto genera que las oficinas de transferencia de tecnología de las universidades puedan ampliar las posibilidades de asociación y los beneficios mutuos (Şendoğdu \& Diken, 2013).

Fiaz (2013) enlista una variedad de factores relacionados con la CUE, entre los que se encuentran el pago por servicios, la categoría de la innovación, el tamaño de la empresa y su ubicación, las capacidades y competencias de cada sector, el apoyo del Estado y los ya mencionados lazos de colaboración previa y la confianza. También está el factor distancia geográfica que, junto con los anteriores, puede afectar de manera positiva o negativa dicha colaboración.

Lo anterior es consistente con los resultados de Petruzzelli (2011), quien 
enfoca su atención en tres factores: parentesco tecnológico, lazos de colaboración previa y distancia geográfica. Este autor sugiere que para que colaboren con éxito las universidades y las empresas se requiere que puedan compartir un cierto nivel de competencias y capacidades similares. Sin embargo, no debe existir demasiada similitud, ya que el desarrollo de innovaciones requiere cuerpos distintos y complementarios de conocimiento. La colaboración previa puede ser la base de la confianza entre socios académicos e industriales y la distancia geográfica, por su parte, no debe considerarse una restricción. En Europa, por ejemplo, existe una importante cantidad de proyectos entre universidades y empresas que se distribuyen a lo largo del continente. Esto que permite inferir que aunque las organizaciones empresariales y educativas estén ubicadas en diversas latitudes, es posible superar de manera conjunta las carencias relacionadas con el carácter limitado de las externalidades del conocimiento.

El papel tradicional de las universidades se ha modificado. Estas se han visto obligadas, entre otras acciones, a incrementar sus actividades de investigación para adaptarse a los constantes cambios en el proceso de innovación y las demandas del sistema económico y social (Slaughter
\& Rhoades, 2004). Para algunos investigadores, esto ha requerido un cambio estructural y una modernización de las habilidades gerenciales y organizativas de las universidades (European Commission, 2008). Desde entonces, según Etzkowitz \& Leydesdorff (2000), ha habido una creciente presión sobre las universidades y sus científicos para que produzcan investigación que sea valiosa para la industria y para que intensifiquen su interacción con otros agentes económicos fuera de su "torre de marfil". Ello permitiría que conocieran los problemas que enfrenta la industria (Gibbons et al., 1994).

También hay aspectos identificados en el ámbito empresarial que deben examinarse, como el hecho que sus representantes creen saber más y tener la solución a muchos problemas. Por esta razón muchos suelen cuestionar los programas de formación de las universidades o no son sensitivos a los requerimientos de los mismos y, en particular, algunos de ellos son reacios a contribuir financieramente en proyectos conjuntos, sobre todo en las pequeñas empresas, en razón de sus limitantes en materia de recursos. Esto reduce la posibilidad de que su personal se especialice.

Múltiplesempresas, además, noacostumbran a comprometerse y dejan en 
manos de las universidades tareas vitales como la recaudación de fondos. Esto con el pretexto que compete al Estado apoyar la investigación, algo que ya no es posible, dado el actual contexto caracterizado por restricciones al gasto público en no pocos países, incluso en los más desarrollados.

El financiamiento es un factor clave para el fomento de la interacción academia-industria. Este puede depender de factores como la composición de la industria local, la disposición de una masa crítica de empresas en el área donde se ubican las universidades e iniciativas políticas relacionadas con la financiación pública y el aumento de la financiación por parte de la industria (Calderini, Franzoni, \& Vezzulli, 2007). De ahí la importancia de examinar las medidas adoptadas en algunos países, como referente para el diseño de políticas públicas que estimulen la CUE.

Por ejemplo, Fiaz (2013) menciona la experiencia de China, en donde la CUE ha ayudado a las pequeñas y medianas empresas a reducir esfuerzos y costos en $\mathrm{I}+\mathrm{D}$ para conseguir ventajas competitivas. Esto ha sido posible gracias a los apoyos e incentivos del gobierno y a los líderes industriales, quienes han confiado en las universidades para compartir proyectos. A pesar de que en China las industrias son consideradas ac- tores protagónicos en el crecimiento económico, mientras la academia es vista como un actor secundario, las evidencias muestran que las universidades han apoyado a la industria para lograr innovaciones, tener un personal competente y contar con una actualizada base científica. Esto es debido a factores que favorecen y alientan dicha colaboración, como la tendencia creciente en $\mathrm{I}+\mathrm{D}$, la existencia de incentivos, la disposición a compartir costos, la aceptación de profesores y alumnos de esta estrategia, así como la estabilidad política, el apoyo del gobierno, la tendencia innovadora, entre otros (Fiaz, 2013).

\subsection{Consideraciones sobre el concepto de CUE}

La academia y la industria trabajan juntas cada vez más. Sin embargo, no ha sido fácil establecer mecanismos de entendimiento mutuo, ni menos lograr que el gobierno respalde proyectos de CUE. Este puede considerarse, en realidad, un fenómeno complejo. Tanto es así que el concepto en sí mismo puede variar en razón de la existencia de una diversidad de enfoques. Para Lee (2000), en particular, se trata de un experimento social en el sistema de innovación de una nación y la sostenibilidad de la experiencia colaborativa se centra en resultados reales, 
esto es, en una relación 'gana-gana' entre universidades y empresas.

La CUE puede detonarse a partir de una actividad básica de las universidades (la capacitación), pero hay una percepción entre los empresarios de que los recursos humanos producidos por las instituciones de educación superior están por debajo de los requerimientos de la industria. Esto debido a su falta de aprendizaje experimental, a su aparente carencia de saberes prácticos. Lo anterior debe ser visto por las universidades no como una amenaza, sino como una oportunidad para acercarse a las industrias a fin de que los estudiantes, entre otros beneficios, puedan adquirir experiencia en ambientes reales antes de graduarse. Para Shin, Lee, Ahn y Jung (2013) la CUE puede generarse también, por ejemplo, si se amplía el periodo de capacitación en la industria. Ello como un primer paso en el acercamiento que puede propiciarse entre ambos actores y facilitar la realización de futuros proyectos conjuntos entre ellos.

Lo anterior facilitaría la formación de confianza, la cual, como se mencionó previamente, reduce las barreras a la CUE y contribuye al entendimiento de similitudes y diferencias entre las partes, esto, además de crear condiciones para llevar a cabo proyectos de mayor envergadura.Hem- mert, Bstieler y Okamuro (2014), en relación con este aspecto, encontraron que la reputación de los socios y el comportamiento exitoso son importantes para la formación de la confianza, en particular, en países emergentes, en donde la mayoría de las empresas y universidades tiene aún poca experiencia en materia de colaboración. Ello al contrario de lo que sucede en países avanzados, en donde es usual que existan fuertes y maduras redes de cue. Más aún, desde una perspectiva de políticas públicas, los autores sugieren que las redes entre empresas y universidades deberían ser fortalecidas y los socios de la colaboración deberían estar provistos con efectivas salvaguardas contractuales para engrandecer la formación de la necesaria confianza.

Trabajos previos sobre la CUE están enfocados más que nada en países desarrollados, sobre todo Estados Unidos, donde la experiencia al respecto tiene una larga data. Sin embargo, ahora que las naciones del sudeste asiático están emergiendo como importantes líderes económicos, se han elaborado estudios acerca de algunos países de esta región. Todavía son limitados los conocimientos acerca de la formación y la gestión de dicha colaboración en estos contextos. Como resultado, existe un bajo entendimiento acerca de, por ejemplo, 
las diferencias que pueden existir en esta materia entrenaciones orientales y occidentales. Tanto en Occidente como en Oriente, en cualquier caso, se considera que el concepto de CUE se encuentra aún en construcción.

Tal vez una consideración particular que se adapta a la nueva realidad de la CUE, en estos múltiples contextos, es que esta se trata de una "relación de colaboración basada en proyectos de investigación entre universidades y empresas, que tiene como objetivo la generación o transferencia de nuevos productos, tecnologías o procesos" (Hemmert et al., 2014, p. 2). Vale la pena agregar que ella debe estar soportada en la confianza, en la reputación de las partes y en salvaguardas contractuales, pero, sobre todas las cosas, en el apoyo del gobierno.

\subsection{El papel del gobierno en apoyo a la CUE}

Desde los ochenta ha existido un marcado interés por impulsar políticas en materia de I+D, en particular en países desarrollados. Por ejemplo, en Estados Unidos, el Reino Unido y Japón los gobiernos han fortalecido las innovaciones con base en políticas científicas y tecnológicas que promueven la prosperidad económica. Estas naciones lo han hecho, en general, a través de tres medidas básicas: 1) financiando directamente los esfuerzos en $\mathrm{I}+\mathrm{D}$, con becas, préstamos, créditos, etc., 2) apoyando al sector privado con financiamiento e incentivos por la vía de impuestos y 3) usando su poder para crear colaboraciones que extiendan y amplíen los esfuerzos en materia de I+D y, así, lograr que la CUE se dé en el contexto de estos esfuerzos interorganizacionales (Rahm, Kirkland \& Bozeman, 2000).

Aunque en los países mencionados ha diferido la manera de implementar las políticas, y su realidad es por supuesto diferente en razón de sus tradiciones, su cultura, la evolución de sus instituciones y la disposición de recursos, entre otros aspectos, estas comparten el hecho de haber aprendido las unas de las otras. En efecto, todas ellas tiene distintas fortalezas que han servido de algún modo ya sea de inspiración, ejemplo o reto a los demás. Esto les ha permitido usar la experiencia de los otros para llevar a cabo una serie de reformas en sus propios contextos (Rahm et al., 2000).

En los tres países mencionados, además, hay una plena conciencia de que fortalecer la CUE es esencial para mejorar la economía nacional $\mathrm{y}$ de que asuntos relevantes, como el de la propiedad intelectual y la forma de gestionar la colaboración, son 
aspectos fundamentales para lograr una efectiva CUE. Kneller (2003) respalda esto y destaca que, de hecho, la perspectiva comparada puede ser valiosa para la definición de estrategias. Este autor resalta que Estados Unidos, por ejemplo, ha implementado reglamentaciones que permiten a las universidades reclamar en todo el mundo derechos de propiedad sobre invenciones hechas bajo contrato o con apoyo del gobierno. Las universidades, además, pueden usar fondos públicos para contratar personal, demandar a las empresas que paguen costos indirectos asociados con la investigación y los investigadores pueden mejorar sus ingresos vía contratos con la industria. También en Japón se trabaja en la materia, ya que hasta 2004 la mayoría de las universidades tenía un estatus financiero que les prohibía recibir dinero extra. Esto, sin embargo, ha ido cambiando.

En Estados Unidos las modificaciones en el marco legal e institucional fortalecieron las universidades y centros de I+D. Esto impulsó la interacción entre academia y empresa. Se crearon también estímulos fiscales y hubo una mayor inversión en I+D. Como resultado, se impulsó la competitividad empresarial basada en I+D. Para Rahm et al. (2000) la aparición décadas atrás de la CUE y los esfuerzos vigorosos del gobierno fe- deral en Estados Unidos representaron un cambio sustancial en la política de investigación, desarrollo e innovación. Uno de los objetivos primarios fue el de apoyar la colaboración aludida, con la esperanza de aumentar la competitividad internacional de la industria. Por otro lado, las leyes aprobadas y otras iniciativas del gobierno alteraron el entorno de $\mathrm{I}+\mathrm{D}$ en dicha nación.

Cuando se hace un análisis retrospectivo de lo ocurrido en el caso de Estados Unidos se observa que este país, en los años setenta, había perdido parte de los mercados de inventos propios como los automóviles, las copiadoras, las televisoras, etc. Esta situación preocupó al gobierno, quien promulgó nueve legislaciones federales, siendo la más conocida el Bayh-Dole Act, lo cual transfirió a las universidades los derechos de propiedad intelectual, aceleró la transferencia de tecnología, incrementó el patentamiento $\mathrm{y}$, entre otras cosas, atrajo a más estudiantes a las universidades. Ello propició la creación de valor y la generación de nuevos empleos, negocios, productos y servicios. Esto fue posible ya que se crearon, en paralelo, estímulos fiscales y hubo una mayor inversión en $\mathrm{I}+\mathrm{D}$.

De acuerdo con Şendoğdu \& Diken (2013), la Ley Bayh-Dole permitió, 
entre otras cosas, 1) garantizar el reparto de los ingresos derivados de comercializar el resultado de las actividades de I+D y 2) que las universidades se concentren en la investigación aplicada en colaboración con la industria. Este renovado espacio de interacción, de hecho, detona la transferencia de tecnología. Más aún, se entiende hoy que dicha ley ha contribuido a bajar los altos niveles de desempleo e inflación y han surgido miles de empresas. Esta experiencia demuestra, de algún modo, que el éxito de la CUE depende en buena medida de cómo los resultados de la investigación afectan o no el entorno micro y macroeconómico de una nación (Liew et al., 2013).

En el Reino Unido, por su parte, el crecimiento en la gestión institucional de la CUE ha sido tan marcado como el aumento en el volumen de $\mathrm{I}+\mathrm{D}$. Se ha registrado además una mayor participación de las universidades, lo cual es producto de que los gobiernos, surgidos de los dos partidos dominantes, han buscado un mejor uso de los recursos sin necesidad de incrementar la inversión pública. Por esta razón, las universidades ven a la CUE como una fuente adicional de ingresos, basada en conocimientos y servicios ya disponibles (Rahm et al., 2000).

En Japón, la cuE difiere marcadamente de la que es realizada en los
Estados Unidos y el Reino Unido, aunque tiene una historia ciertamente legendaria. Motohashi \& Muramatsu (2012) mencionan que Japón promulgó en los años setenta diversas leyes y se diseñaron políticas públicas soportadas por fondos destinados a promover la CUE. En los noventa una nueva oleada de leyes e iniciativas aumentó el número de patentes, aunque en el proceso se ha asumido el riesgo de que disminuya su calidad. En este país, se considera que para las universidades es esencial la promoción de la CUE, así como la conversión de la inversión pública en I+D y en innovaciones industriales. La investigación conjunta de las universidades con las empresas permite además a las unas lograr una comprensión más profunda respecto a las actividades de las otras.

En China, finalmente, la cuE es una práctica obligatoria. Esta, de hecho, busca convertir a esta nación en el centro de la innovación en el mundo. Por esta razón, el gobierno ha considerado que para lograr los objetivos nacionales estratégicos de mejorar la I+D, la colaboración universidad-empresa es esencial. Esto animó a muchas empresas extranjeras a invertir, se generaron también mayores innovaciones y los líderes empresariales vieron a la CUE como una opción para obtener beneficios de la investigación académica (Fiaz, 2013). 
El gobierno chino, además, anunció un Programa Nacional de Mediano y Largo Plazo 2006-2020, en donde se recomienda que grandes empresas presten más atención a las actividades de $\mathrm{I}+\mathrm{D}$, así como a la creación de institutos de I+D y que ambas partes traten de manera deliberada de establecer una mayor colaboración.

Como puede observarse a partir de lo iniciado, el papel del gobierno es esencial para la relación de CUE, tal como Fiaz (2013) y otros autores han señalado. Este genera una presión positiva para trabajar en $\mathrm{I}+\mathrm{D}$, incrementa la intensidad y efectividad de las acciones cooperativas por parte de los agentes involucrados y propicia que se compartan riesgos involucrados en la colaboración en esta materia. Estos aspectos, en efecto, son determinantes para aumentar la CUE. Estas colaboraciones, por lo demás, incentivan la existencia de un entorno articulado de I+D que facilita el logro de rápidas innovaciones generadas gracias a esfuerzos de investigación académica útiles, eficientes y pertinentes.

Un factor determinante para la CUE es el financiamiento. Fiaz (2013) menciona que Estados Unidos, por ejemplo, tiene treinta y nueve reconocidos fondos federales destinados a la investigación y el desarrollo y que organizaciones internacionales suelen apoyar el financiamiento por parte de la industria. Al respecto, hay una fuerte inclinación de los gobiernos, tanto en países desarrollados como emergentes, hacia destinar fondos para la CUE, lo cual se atribuye, entre otros factores, a la creciente necesidad de mantener al día el entorno empresarial para volver a ser -o seguir siendo- competitivos en los mercados. Para Liew et al. (2013), esto solo puede alcanzase a través de un necesario y suficiente apoyo del gobierno. Esto en la forma de fondos financieros, incentivos fiscales e infraestructura en las universidades, entre otros mecanismos.

\section{METODOLOGÍA}

Este documento presenta resultados de investigación terminada desde la perspectiva analítica, sobre el papel del gobierno en apoyo a la colaboración universidad-empresa. Para ello se realizó un análisis comparativo de las contribuciones de autores relevantes que han abordado el tema, en particular, entre 2007 y 2014 . Se consideraron los artículos y otros textos de investigación que aparecen en bases de datos científicas. Esto con el fin de ubicar los hallazgos y vacíos más importantes y así, con base en los conocimientos de los autores de este artículo, comprender y dar a entender los significados y sentidos 
que se mencionan alrededor del fenómeno estudiado.

Este trabajo aborda de manera directa el supuesto de que el gobierno juega un papel decisivo en la CUE, lo cual evidencia una concordancia con lo planteado al inicio del estudio. En función de lo anterior, la interpretación redactada, que se halla inmersa en cada uno de los subtemas que se desarrollan, comporta argumentos que puntualizan criterios de ética y veracidad científica. Como un resultado adicional de este trabajo analítico se identifican los elementos que deben considerar los gobiernos para fortalecer la colaboración universidad-empresa. Estos se presentan en la siguiente sección.

\section{RESULTADOS}

Desde una perspectiva comparada no es fácil hablar de tendencias similares en la actuación de los gobiernos en torno a la CUE y, en general, respecto de sus políticas en materia de ciencia y tecnología. Sin embargo, sí es posible identificar aspectos relacionados con la CUE y con la imprescindible participación del gobierno.

Los resultados del trabajo realizado permitieron establecer diez elementos que es necesario considerar para fortalecer la CUE. Estos se presentan en la tabla 1.
Tabla 1. Elementos para fortalecer la CUE, con la participación del gobierno

\begin{tabular}{|l|}
\hline Revisión del marco legal e institucional \\
\hline Evaluación de experiencias exitosas \\
\hline Establecimiento de salvaguardas \\
\hline Mejoramiento de los recursos humanos \\
\hline Diseño de esquemas de financiamiento \\
\hline Modernización de la infraestructura \\
\hline Apoyos de —y para— las empresas \\
\hline Respaldo a la investigación \\
\hline Impulso a la transferencia de tecnología \\
\hline Promoción de la cuE \\
\hline
\end{tabular}

Fuente: Elaboración propia.

Los elementos mencionados en la tabla inciden en la cuE y están relacionados con el papel que tienen que asumir en particular los gobiernos. Esto independientemente de la realidad de cada país, de la presencia de disímiles niveles de desarrollo o de las acciones que se hayan tomado en distintos tiempos y circunstancias, así como de cualquier tipo de motivaciones.

\section{DISCUSIÓN}

Con relación a los diez elementos identificados para fortalecer la CUE, que deben considerar los gobiernos ya sean federales, estatales o municipales, la fidelidad hacia la lógica de la realidad observada apunta a que el de mayor significancia es la revisión del marco legal e institucional. 
Esto para mejorar las políticas públicas de colaboración. Los demás elementos son:

- La evaluación de experiencias exitosas, para aprovechar el aprendizaje de otros países en la materia.

- El establecimiento de salvaguardas, que generen confianza entre empresas y universidades.

- El mejoramiento de los recursos humanos, que es un primer paso para atender las necesidades del sector productivo.

- El diseño de esquemas de financiamiento, para apuntalar proyectos conjuntos.

- La modernización de la infraestructura, que respalde las actividades de $\mathrm{I}+\mathrm{D}$.

- Los apoyos de -y para-las empresas, que contemplen incentivos del gobierno, así como una mayor disposición de las empresas a apoyar proyectos de CUE, incluyendo recursos financieros por parte de estas.

- Elrespaldoalainvestigación,que es la clave para el progreso de las universidades.

- El impulso a la transferencia de tecnología, actividad que ha demostrado incrementar la interacción entre las universidades y las empresas.

- La promoción de la CUE, como uno de los propósitos centrales de una política científica y tecnológica.

\section{CONCLUSIONES}

La colaboración universidad-empresa (CUE) es parte de un proceso dinámico y cambiante que es clave para la transferencia de conocimiento. Es un recurso vital no solo para generar innovaciones, sino también para que las empresas puedan ser cada vez más competitivas y las universidades logren adaptarse a los cambiantes requerimientos del sector productivo, lo que contribuye a cerrar la brecha entre la industria y la academia. Este fenómeno tiene mucho que ver con el apoyo que brinden los gobiernos, así como la disposición de participar de las empresas y el impulso que den las universidades a proyectos conjuntos y a otras actividades como la capacitación de recursos humanos o la difusión de tecnologías.

Por lo anterior, resulta determinante la articulación de los tres actores aludidos: empresas, universidades y gobierno, pero, en particular, que este último considere los elementos esenciales que permitan fortalecer la CUE (ver tabla), entre otros, la revisión del marco legal e institucional. En este sentido, y tomando en cuenta la experiencia exitosa de otros países, se puede concluir que la colaboración universidad-empresa no 
debe ser solo una prioridad para los gobiernos, sino también una obligación, razón por la cual es preciso que los investigadores profundicen aún más en el establecimiento, el contenido, la organización y la gestión de la mencionada colaboración, para identificar las mejores prácticas posibles en esta materia.

\section{REFERENCIAS}

Barnes, T., Pashby, I., \& Gibbons, A. (2002). Effective university-industry interaction: A multi-case evaluation of collaborative R\&D projects. European Management Journal, 20(3), 272-285.

Bhattacharya, S., \& Arora, P. (2007). Industrial linkages in Indian universities: What they reveal and what they imply? Scientometrics, 70(2), 277-300.

Bjerregaard, T. (2010). Industry and academia in convergence: Microinstitutional dimensions of R\&D collaboration. Technovation, 30, 100-108.

Bodas Freitas, I. M., Argou, R. \& Mirra de Paula, E. (2013). University-industry collaboration and innovation in emergent and mature industries in new industrialized countries. Research Policy, 42, 443-453.

Böhme, G., \& Stehr, N. (Eds.). (1986). The knowledge society: The growing impact of scientific knowledge on social relations. Dordrecht: D. Reidel.

Bruneel, J., D'Este, P., \& Salter, A. (2010). Investigating the factors that diminish the barriers to university-industry collaboration. Research Policy, 39, 858-868.

Calderini, M., Franzoni, C., \& Vezzulli, A. (2007). If star scientists do not patent: The effect of productivity, basicness and impact on the decision to patent in the academic world. Research Policy, 36(3), 303-319.

Carayannis, E. G., \& Campbell, D. F. J. (2012). Mode 3 knowledge production in quadruple helix innovation systems: $21^{\text {st }}$-century democracy, innovation, and entrepreneurship for development. Nueva York - Londres: Springer. Carballo Peña, A., Rodríguez Silva, F. D., \& García Arias, J. M. (2008). La relación universidadempresa: una necesidad para defender el futuro. Ciencias Holguin, XIV(2), 1-11.

Deem, R., Hillyard, S., \& Reed, M. I. (2007). Knowledge, higher education, and the new managerialism: The changing management of UK universities. Oxford - Nueva York: Oxford University Press.

Drucker, P. F. (1996). Landmarks of tomorrow: A report on the new "post-modern" world. New Brunswick: Transaction. 
Etzkowitz, H. (2008). The triple helix: University-industry-government innovation in action. Nueva York: Routledge.

Etzkowitz, H., \& Leydesdorff, L. (2000). The dynamics of innovation: From national systems and "Mode 2" to a triple helix of university-industry-government relations. Research Policy, 29(2), 109-123.

Etzkowitz, H., \& Leydesdorff, L. A. (1997). Universities and the global knowledge economy: A triple helix of university-industry-government relations. Londres Nueva York: Pinter.

European Commission (2008). Diversified funding streams for university-based research: Impact of external project-based research funding onfinancialmanagement in universities. Bruselas: European Commission, DirectorateGeneral for Research.

Fiaz, M. (2013). An empirical study of university-industry R\&D collaboration in China: Implications for technology in society. Technology in Society, 35, 191-202.

Geiger, R. L. (1993). Research and relevant knowledge: American research universities since World War II. Nueva York: Oxford University Press.

Gibbons, M., Limoges, C., Nowotny, H., Schwartzman, S., Scott, P., \& Trow, M. (1994). The new pro- duction of knowledge: The dynamics of science and research in contemporary societies. Londres - Thousand Oaks: SAGE.

Göranzon, B., Hammaren, M., \& Ennals, J. R. (Eds.). (2006). Dialogue, skill and tacit knowledge. Chichester - Hoboken: J. Wiley $\&$ Sons.

Hemmert, M., Bstieler, L. \& Okamuro, H. (2014). Bridging the cultural divide: Trust formation in university-industry research collaborations in the US, Japan, and South Korea. Technovation, 34(10), 605-616.

Hitt, M. A., Ireland, R. D., \& Hoskisson, R. E. (2013). Strategic management: Competitiveness \& globalization $\left(10^{\mathrm{a}}\right.$ ed.). Australia - Mason: South-Western - Cengage Learning.

Hong, W. \& Su, Y. S. (2013). The effect of institutional proximity in non-local university-industry collaborations: An analysis based on Chinese patent data. Research Policy, 42, 454-464.

Jiménez B., M., \& Ramos V., I. (2009). ¿Más allá de la ciencia académica?:Modo2, cienciaposnormal y ciencia posacadémica. Arbor. Ciencia, Pensamiento y Cultura, CLXXXV(738), 721-737.

Khan, G. F., \& Park, H. W. (2012). Editorial: Triple Helix and innovation in Asia using scientometrics, webometrics, and informetrics. Scientometrics, 90, 1-7. 
Kneller, R. (2003). University-industry cooperation and technology transfer in Japan compared with the United States: Another reason for Japan's economic malaise? Journal of International Economic Law, 24, 329-449.

Lai, W-H. (2011). Willingness-toengage in technology transfer in industry-university collaborations. Journal of Business Research, 64, 1218-1223.

Lee, Y. S. (2000). The sustainability of university-industry research collaboration: An empirical assessment. The Journal of Technology Transfer, 25(2), 111-133.

Lee, Y. S. (2012). Strengthening competency linkage to innovation at Korean universities. Scientometrics, 90(1), 219-230. Liew, M. S., Tengku, T. N. \& Lim, E. S. (2012). Strategic and tactical approaches on university-industry collaboration. Procedia Social and Behavioral Sciences, 56, 405-409.

Liew, M. S., Tengku, T. N., \& Lim, E. S. (2013). Enablers in enhancing the relevancy of universityindustry collaboration. Procedia - Social and Behavioral Sciences, 93, 1889- 1896.

Martínez, R., \& Fundación IKERTIA. (2007). La relación universidad-empresa en bizkaia: Un enfoque cualitativo y propuesta de mejora. Revista Escuela de
Administración de Negocios, 61 , 123-125.

McCaffery, P. (2004). The higher education manager's handbook: Effective leadership and management in universities and colleges. Londres - Nueva York: Routledge Falmer.

Mead, N., Beckman, K., Lawrence, J., O'Mary. Parish, C., Unpingco, P., \& Walker, H. (1999). Industry/university collaborations: Different perspectives heighten mutual opportunities. The Journal of Systems and Software, 49, 155-162.

Mockus, A. (2012). Pensar la universidad (2a ed.). Medellín: Fondo Editorial Universidad EAFIT.

Motohashi, K. \& Muramatsu, S. (2012). Examining the university industry collaboration policy in Japan: Patent analysis. Technology in Society, 34, 149-162.

Motoyama, Y. (2014). Long-term collaboration between university and industry: A case study of nanotechnology development in Japan. Technology in Society, 36, 39-51.

Muscio, A., \& Nardone, G. (2012). The determinants of universityindustry collaboration in food science in Italy. Food Policy, 37, 710-718.

Muscio, A., Quaglione, D. \& Scarpinato, M. (2012). The effects of universities' proximity to indus- 
trial districts on university-industry collaboration. China Economic Review, 23, 639-650.

National Research Council (2012). Research universities and the future of America: Ten breakthrough actions vital to our nation's prosperity and security. Washington: The National Academies Press.

Noll, R. G. (Ed.) (1998). Challenges toresearchuniversities. Washington: Brookings Institution Press.

Nonaka, I., \& Takeuchi, H. (1995). The knowledge-creating company: How Japanese companies create the dynamics of innovation. Nueva York: Oxford University Press.

Nonaka, I., \& Takeuchi, H. (2001). Facilitar la creación de conocimiento. México: Oxford University Press.

Nowotny, H., Scott, P., \& Gibbons, M. (2001). Re-thinking science: Knowledge and the public in an age of uncertainty. Cambridge: Polity Press.

Organisation for Economic Co-Operation and Development (OECD). (1996). The knowledge-based economy. Paris: OECD.

Patthareeya, L., \& Tippawan, L. (2012). Knowledge transfer effectiveness of university-industry alliances null. International Journal of Organizational Analysis, 20(2), 128-186.
Petruzzelli, A. M. (2011). The impact of technological relatedness, prior ties, and geographical distance on university-industry collaborations: Ajoint-patent analysis. Technovation, 31, 309-319.

Rahm, D., Kirkland, J., \& Bozeman, B. (2000). University-industry $R \& D$ collaboration in the United States, the United Kingdom, and Japan. (Vol. 1). Dordrecht: Kluwer Academic Publishers.

Ramírez Salazar, M. D. P., \& García Valderrama, M. (2010). La alianza universidad-empresa-Estado: una estrategia para promover innovación. Revista Escuela de Administración de Negocios, 68, 112-133.

Saad, M., \& Zawdie, G. (2011). Theory and practice of the triple helix system in developing countries: Issues and challenges. Nueva York: Routledge.

Salmi, J. (2009). The challenge of establishing world-class universities. Washington: World Bank.

Schilirò, D. (2012). Knowledge-based economies and the institutional environment. Theoretical \& Practical Research in Economic Fields, 3(1), 42-50.

Schwartzman, S. (Ed.). (2008). University and development in Latin America. Rotterdam: Sense.

Şendoğdu,A.A., \& Diken,A. (2013). A research on the problems encountered in the collaboration 
between university and industry. Procedia - Social and Behavioral Sciences, 99, 966-975. Shin, J. S., Lee, K. W., Ahn, J. S., \& Jung, J. W. (2013). Development of internship \& capstone design integrated program for university-industry collaboration. Procedia - Social and Behavioral Sciences, 102, 386-391.

Slaughter, S., \& Rhoades, G. (2004). Academic capitalism and the new economy: Markets, State, and higher education. Baltimore: Johns Hopkins University Press.

Smirnova, Y. V. (2014). Attitudes of companies in Kazakhstan towards knowledge collaboration with universities. Procedia Social and Behavioral Sciences, 109, 639-644.

Soh, P. H., \& Subramanian, A. M. (2014). When do firms benefit from university-industry $R \& D$ collaborations? The implications of firm R\&D focus on scientific. Journal of Business Venturing, 29(6), 807-821.

Teichler, U. \& Arimoto, A. (2014). Teaching and research: A vulnerable linkage? En J.C. Shin, A.
Arimoto, W. K. Cummings \& U. Teichler (Eds.), Teaching and research in contemporary higher education: Systems, activities and rewards (p. 395-401). Nueva York: Springer.

Temple, P. (Ed.). (2012). Universities in the knowledge economy: Higher education organisation and global change. Londres: Routledge.

UNESCO (2005). Hacia las sociedades del conocimiento. París: Ediciones UNESCO.

Viale, R., \& Etzkowitz, H. (2010). The capitalization of knowledge: A triple helix of university-industry-government. Cheltenham - Northampton: Edward Elgar.

Von Krogh, G., Ichijō, K., \& Nonaka, I. (2000). Enabling knowledge creation: How to unlock the mystery of tacit knowledge and release the power of innovation. Oxford - Nueva York: Oxford University Press.

Wang, Q., Cheng, Y., \& Liu, N. (Eds.) (2013). Building worldclass universities: Different approaches to a shared goal. Rotterdam: Sense. 